'Escuela de Obstetricia y

Puericultura, Universidad de Santiago de Chile.

${ }^{2}$ Departamento de

Tocoginecología,

Universidade de Caxias do Sul, Brasil.

aMatrona, Doctor en Salud Pública.

Financiamiento: Proyecto DICYT - Universidad de Santiago de Chile - USACH Presentación de proyecto aprobado en concurso DICYT. La universidad no tuvo influencia en el diseño del estudio, ni en la recolección, análisis, interpretación de los datos o en la preparación o revisión del manuscrito.

Recibido el 1 de julio de 2010, aceptado el 11 de abril de 2011.

Correspondencia a: Dr. Fanny López-Alegría Avda. Ricardo Lyon 504, ap. 210, Providencia, Santiago, Chile. Fono celular: 84729215 E-mail: fanny.lopez@ usach.cl

\section{Síntomas climatéricos y calidad de vida de usuarias de consultorios de atención primaria de salud, Santiago}

\author{
FANNY LÓPEZ-ALEGRÍA ${ }^{1, a}$, DINO ROBERTO SOARES DE LORENZI ${ }^{2}$
}

Background: During menopause the quality of life of women can be seriously deteriorated and influenced by lifestyles. Aim: To assess the prevalence of menopausal symptoms and their relationship with lifestyles and quality of life. Material and Methods: The Menopause Rating Scale (MRS), a survey that measures menopausal symptoms and has questions related to lifestyles, was applied to 1023 women aged 54 \pm 6 years (range 45 to 64 years), consulting in primary health care clinics. A multiple linear regression analysis was used to evaluate the association between menopausal symptoms and lifestyle. Results: Fifty six percent of women did not have a paid work, 64\% were nonsmokers and 90\% did not perform any physical exercise. The most frequent menopausal symptoms referred were muscle and joint ailments (85\%) followed by mental and physical exhaustion (80\%). Sedentary women, smokers or those who did not to have time for leisure activities showed worst quality of life scores. Conclusions: Postmenopausal women with unhealthy lifestyles had the lower quality of life scores and more menopausal symptoms.

(Rev Med Chile 2011; 139: 618-624).

Key words: Life style; Menopause; Quality of life.

L a población chilena en las últimas décadas ha aumentado su expectativa de vida, produciéndose un incremento en el número de mujeres que alcanza la etapa del climaterio. Esta situación se evidencia en las mujeres de edades entre 45 y 64 años que han aumentado de 1.079.552 a 1.428 .490 a nivel nacional y de 447.258 a 836.449 a nivel de la Región Metropolitana, de acuerdo con los censos de 1992 y 2002. Esto significa un incremento de $87 \%$ de mujeres en fase de climaterio en esta última región ${ }^{1,2}$.

El climaterio es un proceso fisiológico caracterizado por una disminucion progresiva de la secreción de estradiol que provoca, además de irregularidades menstruales, manifestaciones de diverso orden en el ámbito somático vegetativo, psicológico y urogenital. Según Arteaga en Chile y De Lorenzi en Brasil, esta variedad de síntomas y su intensidad pueden modificarse de acuerdo a factores sociales, culturales y físicos ${ }^{3,4}$. Si, además de estas variables, consideramos las diferentes percepciones de cada mujer con respecto a sus propios síntomas, es necesario uniformar criterios para evaluar estos síntomas. En respuesta a esta situación, se creó la escala Menopause Rating Scale (MRS), la que permite obtener mediciones de la calidad de vida específica para el período del climaterio, y a su vez, comparar, a nivel epidemiológico, las distintas poblaciones de mujeres ${ }^{5-7}$.

Con respecto al manejo clínico de la mujer con esta sintomatología, tradicionalmente se remitía al alivio de los síntomas vasomotores y prevención de osteoporosis a través de la terapia de reposición hormonal. Esto trajo como consecuencia un proceso de medicación intenso, lo que acentuó la percepción negativa de la menopausia ${ }^{8}$. Sin 
embargo, esta prescripción medicamentosa tuvo un proceso de revisión a consecuencia del estudio WHI (Women's Health Initiative), publicado en el año $2002^{9}$.

A partir de este estudio, la asistencia a la mujer climatérica pasó a tener como eje directriz la calidad de vida. Así, actualmente, al proponer una atención integral a la mujer climatérica, si bien no se dejan de considerar los beneficios de la terapia hormonal, se prefieren medidas menos invasivas, como el estímulo a comportamientos saludables $^{4,10}$.

En esta línea, autores como De Lorenzi, Oliveira y Aldrighi, en la actual década, han investigado diferentes poblaciones de mujeres en fase de climaterio, concluyendo que la sintomatología propia de este período está relacionada con sus hábitos de vida, tales como el consumo de cigarrillos, la actividad física y recreativa, entre otras ${ }^{4,11,12}$.

En Chile, se realizó la Encuesta Nacional de Calidad de Vida y Salud 2000 y la Encuesta Nacional de Salud 2003, en las cuales se abordaron los temas del sedentarismo, la obesidad, el consumo de tabaco y la calidad de vida propiamente tal; obteniendo como resultado una alta prevalencia de hábitos perjudiciales para la salud ${ }^{13,14}$. Frente a esta situación, las autoridades implementaron los "Objetivos Sanitarios para la Década 2000-2010", en los que se encuentran políticas de salud dirigidas a las mujeres de esta fase etaria ${ }^{15,16}$.

Con la gran cantidad de población femenina que existe actualmente en nuestro país en fase de climaterio y la alta prevalencia de hábitos de vida que no promueven la salud, hacen necesario el presente estudio, que pretende determinar la prevalencia de los síntomas menopáusicos y la posible relación con los hábitos de vida que tienen las mujeres climatéricas beneficiarias del Servicio de Salud Metropolitano de Santiago de Chile, utilizando la escala Menopause Rating Scale (MRS).

\section{Material y Métodos}

Es un estudio epidemiológico de prevalencia y asociación, que se basa en un diseño observacional de corte transversal con alcance descriptivo y correlacional. Con el fin de asegurar la representatividad de todos los estratos socio-geográficos, se consideraron las seis áreas de salud en que se divide la Región Metropolitana de Santiago de
Chile, según el Ministerio de Salud. Así, se realizó un muestreo estratificado en estas 6 áreas, resultando elegidos en total 11 consultorios de atención primaria de salud. Posteriormente, se seleccionó el número de mujeres por consultorio, aplicándose un muestreo aleatorio simple, proporcional al tamaño de cobertura de la población de mujeres beneficiarias de cada consultorio, resultando en total 1.023 mujeres entrevistadas en el $2^{\circ}$ semestre del año 2008. El cálculo del tamaño de muestra se realizó considerando 95\% de nivel de confianza, $5 \%$ de error aceptable y una frecuencia del factor de estudio de $69 \%$.

Los criterios de inclusión fueron: mujeres de 45 a 64 años con estado de salud normal, definido según el National Center for Health Statistic ${ }^{17}$. Los criterios de exclusión fueron mujeres con patologías crónicas como diabetes mellitus e hipertensión arterial, usuarias de medicamentos antidepresivos, ansiolíticos o terapia de reposición hormonal. Frente a la posibilidad de presentar dificultad en la interpretación o llenado del cuestionario, se excluyeron las mujeres con problemas de visión, déficit mental o analfabetas.

Una vez elegidas las mujeres de acuerdo a los criterios de selección, estas fueron informadas de los objetivos del estudio y si estaban de acuerdo con participar, se les solicitaba la firma del consentimiento informado.

El instrumento de recolección de datos fue un cuestionario autoaplicado que incluyó las siguientes variables: edad (en años), estado menopáusico definido según historia menstrual de las mujeres en los últimos 12 meses, estableciéndose para el actual estudio, el estado de pre-menopausia para aquellas mujeres que referían ciclos menstruales en los últimos 12 meses y postmenopausia para aquellas mujeres que no han tenido menstruación en el último año.

Como hábitos fueron considerados, en primer lugar, el tabaquismo, definido como el consumo de cigarrillos en los últimos 3 meses (fuma o no fuma); en segundo lugar, el ejercicio físico regular, el que entendemos como la práctica de alguna actividad física 3 o más veces a la semana, por al menos 30 minutos (practica o no practica) ${ }^{14}$; en tercer lugar, las actividades recreativas, que comprendemos como el conjunto de prácticas de índole social, que se realizan en el tiempo libre de trabajo y que otorguen un disfrute ${ }^{18}$; en cuarto lugar las actividades domésticas que se entienden 
como la práctica de tareas o labores dentro el hogar y por último la ocupación remunerada, considerada como el ejercicio de una actividad laboral a cambio de un sueldo.

Estos actividades fueron evaluados dentro de los 3 últimos meses y con respuestas dicotómicas (sí o no)

Para la evaluación de los síntomas climatéricos y calidad de vida se utilizó la versión en español de la escala Menopause Rating Scale y validada en Chile por Aedo et a ${ }^{19}$. La escala MRS está compuesta por once síntomas, agrupados en tres dominios: somático-vegetativo, psicológico y urogenital. El dominio somático vegetativo incluye los bochornos, molestias cardiacas, dificultades del sueño, molestias musculares y articulares. Los síntomas psicológicos agrupan la depresión, irritabilidad, ansiedad, agotamiento físico y mental. Por último, los síntomas urogenitales, incluyen los problemas sexuales, de vejiga y sequedad vaginal. La medición de la intensidad de cada síntoma de la escala MRS fue de tipo escalonamiento de Likert ${ }^{20}$. Cada síntoma es autoevaluado por la mujer con un puntaje de 0 a 4 según el grado de intensidad: $0=\sin$ molestia; $1=$ molestia leve; $2=$ molestia moderada; $3=$ molestia severa; $4=$ molestia intolerable. Para la evaluación por dominios se consideró el siguiente puntaje: en los dominios somático-vegetativo y psicológico: $0=$ sin molestia; 1 a $4=$ molestia leve; 5 a $8=$ molestia moderada; 9 a $12=$ molestia severa; 13 a $16=$ molestia intolerable. Para el dominio urogenital se consideró $0=\sin$ molestia; 1 a $3=$ molestia leve; 4 a $6=$ molestia moderada; 7 a $9=$ molestia severa $; 10$ a $12=$ molestia intolerable. Para la suma total de la escala, la cual arrojaría la intensidad sintomática de cada mujer, se consideró $0=\sin$ molestia; 1 a 11 = molestia leve; 12 a 22 = molestia moderada; 23 a $33=$ molestia severa; 34 a $44=$ molestia intolerable.

\section{Estadística}

Los datos recolectados fueron analizados electrónicamente a través del programa estadístico SPSS (versión 14) y se calcularon las frecuencias simples, medias, medianas y desvíos padrón. Para el análisis de las variables independientes en el grado de comprometimiento de la calidad de vida se utilizó el análisis por regresión linear múltiple, teniendo como variable dependiente los puntajes globales de la escala MRS. Para ser incluidas en el modelo de regresión, las variables fueron dicotomizadas, recibiendo valores de " 0 " y " 1 ", conforme la ausencia o presencia del factor de estudio (dummy variables). En todos los análisis realizados se aceptó un nivel de significancia de 5\%.

\section{Resultados}

El perfil demográfico de las 1.023 pacientes que participaron en la investigación fue el siguiente: edad promedio de 54,2 $\pm 6,0$, con una moda de 50 años y con edad mínima de 45 y máxima de 64 años, los que son rangos de edades extremas de acuerdo a los parámetros etarios definidos por el programa de climaterio. En relación al estado menopáusico, $66,9 \%$ de ellas eran postmenopáusicas y en cuanto a sus hábitos, gran parte de estas mujeres, referían no fumar $(64,5 \%)$, no practicar ejercicios físicos regularmente $(90,2 \%)$, no tener ocupación remunerada al momento de la entrevista $(56 \%)$, dedicarse a actividades domésticas por lo menos una vez a la semana $(97,1 \%)$ y permitirse horarios para practicar actividades recreativas 0 de descanso $(94,0 \%)$, como ver televisión, visitar amistades o parientes, dormir y tejer o coser (Tabla 1).

En cuanto a los síntomas climatéricos, la mayoría de la mujeres sufren de molestias musculares y articulares $(84,5 \%)$ y agotamiento físico y mental $(80,4 \%)$; siendo los síntomas de menor frecuencia los del dominio urogenital, con un porcentaje inferior a $60 \%$, para cada uno de los 3 síntomas que componen este dominio (Tabla 2).

A través de la escala MRS se evaluó la severidad de la sintomatología climatérica, en un primer lugar, por síntomas, obteniendo el mayor puntaje las molestias musculares y articulares $(1,82)$ y el menor, la sequedad vaginal $(0,84)$, lo que se considera como un rango de molestias de intensidad leve. Al evaluar por dominios de síntomas, observamos que el psicológico es el más afectado $(5,74)$, definido como molestias de intensidad moderada. Finalmente, la evaluación global reveló un compromiso moderado de la calidad de vida de las mujeres $(13,86)$, medido a través de los índices totales de la escala MRS (Tabla 3).

La sintomatología climatérica observada en este estudio se correlacionó con las variables estado menopáusico, práctica regular de ejercicio físico, tabaquismo y tiempo libre para activi- 
Tabla 1. Distribución del perfil sociodemográfico y hábitos de vida de mujeres en fase de climaterio

\begin{tabular}{|c|c|c|}
\hline Variables & $\mathbf{n}$ & $\%$ \\
\hline $\begin{array}{r}\text { Edad (años) } \\
\text { - } 45-49 \\
\text { - } 50-54 \\
\text { - } 55-59 \\
\text { - } 60-64\end{array}$ & $\begin{array}{l}264 \\
289 \\
219 \\
251\end{array}$ & $\begin{array}{l}25,8 \\
28,3 \\
21,4 \\
24,5\end{array}$ \\
\hline $\begin{array}{l}\text { Consumo de cigarrill } \\
\text { - No fuma } \\
\text { - Sí fuma }\end{array}$ & $\begin{array}{l}660 \\
363\end{array}$ & $\begin{array}{l}64,5 \\
35,5\end{array}$ \\
\hline $\begin{array}{l}\text { Ejercicio físico regula } \\
\text { - No practica } \\
\text { - Si practica }\end{array}$ & $\begin{array}{l}923 \\
100\end{array}$ & $\begin{array}{r}90,2 \\
9,8\end{array}$ \\
\hline $\begin{array}{l}\text { Tiempo semanal para } \\
\text { recreativas } \\
\text { - No dedica } \\
\text { - Si dedica }\end{array}$ & $\begin{array}{r}62 \\
961\end{array}$ & $\begin{array}{r}6,0 \\
94,0\end{array}$ \\
\hline $\begin{array}{l}\text { Practica semanal de a } \\
\text { domesticas } \\
\text { - No } \\
\text { - Sí }\end{array}$ & $\begin{array}{r}27 \\
996\end{array}$ & $\begin{array}{r}2,9 \\
97,1\end{array}$ \\
\hline $\begin{array}{l}\text { Ocupación remunera } \\
\text { - No tiene } \\
\text { - Si tiene }\end{array}$ & $\begin{array}{l}573 \\
450\end{array}$ & $\begin{array}{l}56,0 \\
44,0\end{array}$ \\
\hline $\begin{array}{l}\text { Estado menopáusico } \\
\text { - Pre-menopausa } \\
\text { - Pos-menopausa }\end{array}$ & $\begin{array}{l}339 \\
684\end{array}$ & $\begin{array}{l}33,1 \\
66,9\end{array}$ \\
\hline Total & 1.023 & 100,0 \\
\hline
\end{tabular}

dades recreativas. De esta manera, las mujeres postmenopáusicas, fumadoras, sin tiempo para actividades recreativas o sedentarias tuvieron los peores puntajes de calidad de vida y sintomatología climatérica, en general, más severa (Tabla 4).

\section{Discusión}

Los resultados de nuestra investigación en el aspecto de los hábitos de vida perjudiciales a la salud, se condicen con los obtenidos en la Encuesta Calidad de Vida y Salud 2006, en la cual se enuncia que $38 \%$ de las mujeres chilenas de 45 a 64 años han fumado por lo menos 1 cigarrillo en el último mes, y $93 \%$ de estas mujeres son sedentarias ${ }^{21}$. Al compararlo con nuestra población de estudio, $35,5 \%$ de las mujeres son fumadoras y $90,2 \%$ son sedentarias. Estos resultados evidencian la similitud porcentual que tienen nuestros hallazgos con respecto a la misma población femenina nacional de años anteriores. Es importante destacar que estos porcentajes fueron antecedentes que impulsaron al Ministerio de Salud de Chile, a establecer las metas nacionales en salud a través de los Objetivos Sanitarios para la Década 2000-2010, que proyectaron descender los índices a 30\% en el consumo de tabaco y a $84 \%$ en el sedentarismo en la población general para el año $2010^{15}$.

Otro de los factores considerado indicadores de calidad de vida y salud, es el trabajo, según la

Tabla 2. Distribución de la frecuencia y porcentajes de la intensidad de la sintomatología climatérica medidos por Menopause Rating Scale (MRS)

\begin{tabular}{|lccccc|}
\hline Intensidad / Síntomas & Ausentes & Leve & Moderado & Severo & Intolerable \\
\hline Bochornos & $369(36,1 \%)$ & $324(31,7 \%)$ & $187(18,2 \%)$ & $100(9,8 \%)$ & $43(4,2 \%)$ \\
\hline Molestias al corazón & $402(39,3 \%)$ & $326(31,9 \%)$ & $223(21,8 \%)$ & $66(6,4 \%)$ & $6(0,6 \%)$ \\
\hline Dificultades en el sueño & $294(28,7 \%)$ & $307(30,0 \%)$ & $246(24,1 \%)$ & $148(14,5 \%)$ & $28(2,7 \%)$ \\
Molestias musculares y articulares & $158(15,5 \%)$ & $256(25,0 \%)$ & $285(27,9 \%)$ & $257(25,1 \%)$ & $67(6,5 \%)$ \\
\hline Estado de ánimo depresivo & $278(27,2 \%)$ & $266(26,0 \%)$ & $305(29,8 \%)$ & $145(14,2 \%)$ & $29(2,8 \%)$ \\
\hline Irritabilidad & $254(24,8 \%)$ & $303(29,6 \%)$ & $282(27,6 \%)$ & $145(14,2 \%)$ & $39(3,8 \%)$ \\
Ansiedad & $261(25,5 \%)$ & $304(29,7 \%)$ & $279(27,3 \%)$ & $153(15,0 \%)$ & $26(2,5 \%)$ \\
\hline Cansancio físico y mental & $201(19,6 \%)$ & $326(31,9 \%)$ & $298(29,1 \%)$ & $175(17,1 \%)$ & $23(2,3 \%)$ \\
\hline Problemas sexuales & $444(43,3 \%)$ & $207(20,2 \%)$ & $193(18,9 \%)$ & $158(15,5 \%)$ & $21(2,1 \%)$ \\
\hline Problemas con la orina & $416(40,7 \%)$ & $310(30,3 \%)$ & $199(19,4 \%)$ & $81(7,9 \%)$ & $17(1,7 \%)$ \\
\hline Sequedad vaginal & $491(48,0 \%)$ & $319(31,2 \%)$ & $135(13,2 \%)$ & $59(5,8 \%)$ & $19(1,8 \%)$ \\
\hline
\end{tabular}


Tabla 3. Puntaje de los síntomas climatéricos medidos por Menopause Rating Scale (MRS) presentados por las mujeres

\begin{tabular}{|llcc|}
\hline $\begin{array}{l}\text { Dominios } \\
\text { (MRS) }\end{array}$ & Síntomas & $\begin{array}{c}\text { Puntaje de síntomas } \\
\text { (media } \pm \text { DE) }\end{array}$ & $\begin{array}{c}\text { Puntaje de dominios } \\
\text { (media } \pm \text { DE) }\end{array}$ \\
\hline Somático & Bochornos & $1,00 \pm 0,7$ & $5,14 \pm 3,1$ \\
& Molestias cardiacas & $0,99 \pm 1,0$ & $1,33 \pm 1,1$ \\
Dificultades sueño & Molestias musculares y articulares & $1,82 \pm 1,1$ & $5,74 \pm 3,3$ \\
& Estado de ánimo & $1,40 \pm 1,1$ & \\
& Irritabilidad & $1,43 \pm 1,1$ & $2,98 \pm 2,2$ \\
& Ansiedad & $1,40 \pm 1,1$ & $1,86 \pm 1,1$ \\
\hline
\end{tabular}

Tabla 4. Factores asociados a la severidad de la sintomatología climatérica y calidad de vida (MRS-puntaje total)

\begin{tabular}{|lrr|}
\hline Variables & b & P \\
$\begin{array}{l}\text { Espacio semanal para actividades } \\
\text { recreativas }\end{array}$ & $-0,066$ & 0,03 \\
\hline $\begin{array}{l}\text { Ejercicio físico regular } \\
\text { Tabaquismo }\end{array}$ & $-0,135$ & $<0,01$ \\
$\begin{array}{l}\text { Estado menopáusico } \\
\text { (pos-menopausia) }\end{array}$ & 0,065 & 0,03 \\
\hline
\end{tabular}

Análisis por regresión linear múltiple y ajustado para las variables edad, tabaquismo, actividades recreativas, estado menopáusico (pos-menopausia), ocupación remunerada y actividades domésticas.

Encuesta de Calidad de Vida y Salud $2006^{21,22}$, indicando que $46 \%$ de las mujeres de 45 a 64 años tienen ocupación laboral, porcentaje similar al observado en nuestras mujeres de estudio (44\%).

Con respecto a la sintomatología, al realizar una revisión de la literatura, se encuentran que los estudios publicados utilizando la escala MRS, revelan que entre los continentes, América Latina aparece con los puntajes globales más altos $(10,4)^{6}$. Estos planteamientos se reafirman en un estudio multicéntrico $(2008)^{23}$, el cual ubica a Chile, entre los 12 países latinoamericanos estudiados, como el poseedor de la peor calidad de vida para el grupo etario climatérico, obteniendo un puntaje total de 16,3; resultados que también obtiene Prado et al $(2008)^{24}$, en nuestro país. De esta manera, al contrastar los resultados anteriores con nuestro estudio, evidenciamos un puntaje de menor compromiso $(13,8)$ en nuestras mujeres.

En el análisis de los resultados de la relación entre estos síntomas climatéricos y los hábitos de vida de las mujeres de nuestro estudio, estos concuerdan con los estudios de algunos autores como De Lorenzi y cols (2005), quienes evaluaron la sintomatología referida por 254 mujeres postmenopáusicas usuarias del Consultorio de Climatério de la Universidad de Caxias do Sul, Brasil. El análisis multivariado reveló que las mujeres con historia de actividad física regular y con una percepción o actitud positiva frente a la menopausia manifestaban una sintomatología climatérica significativamente menos severa, reforzando la influencia de factores psicosociales y culturales ${ }^{25}$. Además, Oliveira y col (2001), concluyó que las mujeres climatéricas fumadoras, asistentes al Programa de Salud de Cuiabá, Brasil, presentaban más síntomas vasomotores que las no fumadoras ${ }^{11}$. Por su parte, Polisseni et al (2009), 
observaron una relación significativa entre la presencia de síntomas climatéricos moderados y las alteraciones de humor ${ }^{26}$. Además, otros estudios (Nelson et al 2008) han demostrado que la práctica regular de ejercicios físicos disminuye los niveles de ansiedad y depresión durante la menopausia ${ }^{27}$.

Sin embargo, también existen otras investigaciones que no relacionan la prevalencia de los síntomas del climaterio con los hábitos de vida como en nuestro estudio. En un estudio realizado a un grupo de mujeres de Baltimore (2007), se concluyó que la actividad física frecuente se asociaba con una mayor frecuencia y severidad de bochornos ${ }^{28}$. Además, Vega et al (2007) concluyó, a partir del análisis de una población de mujeres mexicanas, que el tabaquismo y el ejercicio físico eran factores que no presentaban asociación estadísticamente significativa con los síntomas menopáusicos ${ }^{29}$. Por último, en Australia, Van Poppel MN (2008) concluyó que la actividad física no se asocia con los síntomas de la menopausia ${ }^{30}$.

En conclusión, la alta prevalencia e intensidad, en general, más severa de estos síntomas, produce un compromiso moderado de la calidad de vida de las mujeres; lo que junto a los hábitos de vida no saludables de este grupo etario: tabaquismo y sedentarismo, producen un aumento en la severidad de la sintomatología climatérica, lo que se ve reflejado en un puntaje global de 13,8 en la escala MRS. Es importante destacar que nuestros hallazgos confirman un avance mínimo de los indicadores de tabaco y sedentarismo definidos por el Minsal, para la actual década. Además los presentes resultados colaboran con la ausencia de información que existió en la evaluación parcial del alcance de los Objetivos Sanitarios del Minsal, respecto de las metas para mejorar de la calidad de vida de las mujeres en etapa de climaterio.

Agradecimientos: a Bárbara Rivera por la revisión de la redacción del manuscrito.

\section{Referencias}

1. Instituto Nacional de Estadísticas, Chile. Informe demográfico de Chile Censo 1992. 2a. ed. actualizada. Santiago, Chile: INE, 1997. 49 p.

2. Departamento de Estadísticas e Información de SaludDEIS. Ministerio de Salud. Población Censo 2002.

3. Arteaga E, Bianchi M. Fisiología del climaterio y meno- pausia. En: Pérez Sánchez A. Ginecología. 3a ed. Santiago, Chile: Editorial Mediterráneo Ltda.; 2003. p. 179-85.

4. De Lorenzi DRS, Chada E, Saciloto B, Padilha I. Fatores associados á qualidade de vida após menopausa. Rev Assoc Med Bras 2006; 52: 312-7.

5. Heinemann LA, Potthoff P, Schneider HP. International versions of the Menopause Rating Scale (MRS). Health Qual Life Outcomes 2003; 1: 28-34.

6. Heinemann K, Ruebig A, Potthoff P, Schneider H, Strelow F, Heinemann L, et al. The Menopause Rating Scale (MRS) scale: A methodological review. Health Qual Life Outcomes 2004; 2: 45-52.

7. Dinger J, Zimmermann T, Heinemann L, Stoehr D. Quality of life and hormone use: new validation results of MRS scale. Health Qual Life Outcomes 2006; 2: 32-6.

8. Mendonça EAP. Representações médicas e de gênero na promoção da saúde no climatério/menopausa. Ciênc saúde coletiva 2004; 9:155-66.

9. De Lorenzi DRS, Baracat EC. Climatério e qualidade de vida. Femina 2005; 33: 809-903.

10. Kakkar V, Kaur D, Chopra K, Kaur A, Kaur IP. Assessment of the variation in menopausal symptoms with age, education and working/non-working status in north-Indian sub population using menopause rating scale (MRS). Maturitas 2007; 57: 306-14.

11. Oliveira V, Valente J, Madeiros S. Hábitos e estilo de vida em mulheres climatéricas de baixa renda em Cuiabá. Reprod clim 2001; 16: 253-8.

12. Aldrighi J, Alecrin I, Oliveira P. de, Shinomata H. Tabagismo e antecipação da idade da menopausa. Rev Assoc Med Bras 2005; 51: 51-3.

13. Departamento de Epidemiología \& Departamento de Promoción de la Salud. Encuesta Nacional de Calidad de Vida y Salud 2000. Minsal, 2001; p. 49.

14. Ministerio de Salud de Chile. Encuesta Nacional de Salud Chile 2003: Desarrollo del trabajo de campo. 2003.

15. Ministerio de Salud de Chile. Objetivos Sanitarios para la Década 2000-2010: Resumen ejecutivo. El Vigía, Boletín de Vigilancia en Salud Pública de Chile, 2002; 5: 1-12.

16. Departamento de Epidemiología \& Departamento de Promoción de la Salud. Ministerio de Salud de Chile. Resumen ejecutivo Encuesta Nacional de Salud, 2003. El Vigía 2004; 8: 1-19.

17. Brett KM, Chong Y. Hormone replacement therapy: Knowledge and use in the United States. Hyattsville, Maryland: National Center for Health Statistic. 2001.

18. Formiga NS, Ayroza I, Dias L. Escala das atividades de hábitos de lazer: construção e validação em jovens. Psic. 2005; 6: 71-9.

19. Aedo S, Porcille A, Irribarra C. Calidad de vida relacionada con el climaterio en una población chilena de 
mujeres saludables. Rev Chil Obstet Ginecol 2006; 71: 402-9.

20. Berlin Center for Epidemiology and Health Research. Evaluation \& reference values. MRS - the menopause rating scale 2008. Disponible en: www.menopauserating-scale.info/evaluation.htm (Consultado el 29 de abril de 2009).

21. Ministerio de Salud de Chile. II Encuesta Nacional de Calidad de Vida y Salud Chile 2006.

22. Ministerio de Salud de Chile. Objetivos Sanitarios para la Década 2000-2010: Evaluación a mitad de período. Estado de Avance en los objetivos de impacto. 2006; pp 73.

23. Chedraui P, Blümel JE, Baron G, Belzares E, Bencosme A, Calle A, et al. Impaired quality of life among middle aged women: A multicentre Latin American study. Maturitas 2008; 61:323-9.

24. Del Prado M, Fuenzalida A, Jara D, Figueroa R, Flores D, Blumel JE. Evaluación de la calidad de vida en mujeres de 40 a 59 años mediante la escala MRS (Menopause Rating Scale). Rev Med Chile 2008; 136: 1511-7.
25. De Lorenzi DRS, Danelon C, Saciloto B, Padilha Jr I. Fatores indicadores da sintomatología climatérica. Rev Bras Ginecol Obstet 2005; 27: 12-9.

26. Polisseni A, Polisseni F, Fernandes L, Aredes M, Oliveira M. Depressão em mulheres climatéricas: fatores associados. HU Rev, Juiz de Fora 2009; 35: 183-9.

27. Nelson DB, Sammel MD, Freeman EW, Lin H, Gracia CR, Mogul M, et al. Effect of Physical Activity on Menopausal Symptoms among Urban Women. Med Sci Sports Exerc 2008; 40: 50-8.

28. Whitcomb BW, Whiteman MK, Langenberg P, Flaws JA, Romani WA. Physical activity and risk of hot flashes among women in midlife. J Womens Health 2007; 16: 124-33.

29. Vega G, Hernández A, Leo G, Vega J, Escartin M, Luengas $\mathrm{J}$, et al. Incidencia y factores relacionados con el síndrome climatérico en una población de mujeres mexicanas. Rev Chi Obstet Ginecol 2007; 72: 314-20.

30. Van Poppel MN, Brown WJ. Does physical activity help with menopausal symptoms? Menopause 2008; 15: 78 85. 